JOURNAL DE PHYSIQUE IV

Colloque C7, supplément au Journal de Physique III, Volume 4, juillet 1994

\title{
Quantitative photomodulated thermoreflectance studies of germanium and silicon semiconductors
}

\author{
R.E. Wagner and A. Mandelis \\ Photothermal and Optoelectronic Diagnostics Laboratory, Department of Mechanical Engineering, \\ University of Toronto, Toronto, Ontario, Canada, M5S 1A4
}

\begin{abstract}
The frequency response of the photomodulated thermoreflectance (PMTR) signal has been used to characterize various semiconductor samples, including crystalline $\mathrm{Ge} / \mathrm{Si}$, ion-implanted $\mathrm{Ge}$, and amorphous Si. Theoretical modelling has allowed the deconvolution of electron-hole plasma- and thermal-wave contributions to the signal throughout the entire frequency range.
\end{abstract}

\section{INTRODUCTION}

The photomodulated thermoreflectance (PMTR) method [1] has been used to characterize various semiconductor samples, including crystalline $\mathrm{Ge} / \mathrm{Si}$, ion-implanted $\mathrm{Ge}$, and amorphous $\mathrm{Si}$. In particular, the PMTR signal has been measured as a function of the modulation frequency, over the range $10^{3}$ to $1.6 \times 10^{6} \mathrm{~Hz}$, for two different $1 / \mathrm{e}$ pump-beam radii, 5.7 and $23.3 \mu \mathrm{m}$. In all cases, the pump wavelength was $514.5 \mathrm{~nm}$, while the probe wavelength was $632.8 \mathrm{~nm}$. In order to gain insight into the various signal generation mechanisms, each experimental curve was fitted to a theoretical curve. The theoretical data were calculated by taking into account a number of reflectance modulation mechanisms: The change in sample temperature; the variation in the density of free-carriers; and, for the disordered materials, the change in the density of carriers trapped in localized gap-states.

Measurements were performed on six different $\mathrm{Ge}$ and Si samples. For instance, two different Ge samples were studied, a crystalline specimen (c-Ge) and an ion-implanted wafer (im-Ge). The c-Ge wafer had a $<111>$ surface orientation, a resistivity of $15 \Omega \cdot \mathrm{cm}$ (p-type), and a thickness of $200 \mu \mathrm{m}$. The im-Ge wafer was identical to the c-Ge wafer except that it was implanted with $100 \mathrm{keV}$ phosphorus ions to a dose of $10^{16} \mathrm{~cm}^{-2}$. According to published ion-range tables [2], the im-Ge possessed an amorphous damage layer of $0.134 \mu \mathrm{m}$ thickness.

Four different $\mathrm{Si}$ samples were characterized, three crystalline samples and an amorphous thinfilm. Two of the c-Si samples were obtained from a wafer with the following processing history. A $<100>\mathrm{n}$-type wafer with a thickness of $385 \mu \mathrm{m}$ and a resistivity of $100 \Omega \cdot \mathrm{cm}$ was ion-implanted over half of its surface; the implant dose was $10^{15} \mathrm{~cm}^{-2}$ of arsenic ions at $180 \mathrm{keV}$. The wafer was then flash annealed at $950^{\circ} \mathrm{C}$ for $20 \mathrm{~s}$. Following this annealing, the implanted side of the wafer had a new surface-layer resistivity of $10 \Omega \cdot \mathrm{cm}$. Then, two samples were obtained from the Si wafer described above: c-Si1 consisted of non-implanted material, and c-Si2 consisted of implanted and annealed material. The third $c$-Si sample (c-Si3) was p-type, had a $<100>$ surface orientation, and possessed a resistivity of $2.3 \Omega \cdot \mathrm{cm}$. Finally, the a-Si specimen consisted of $0.8 \mu \mathrm{m}$ of amorphous silicon deposited on a c-Si substrate. The a-Si was grown at $570^{\circ} \mathrm{C}$ by a low-pressure CVD process. 


\section{RESULTS AND DISCUSSION}

In order to fit the experimental PMTR data to the theoretical model, the following methodology was employed. First, as far as was possible, a sample's physical properties were obtained from the literature [3], or by independent measurement. For the crystalline samples it was quite straightforward to obtain many of the required material properties; on the other hand, for the amorphous samples it was not possible to obtain many of the required parameters to a great degree of accuracy, due to the fact that electronic transport is not well understood in disordered materials, and because the behavior of a particular material is strongly dependent upon it processing history.

For the crystalline samples, the only material parameters which were not well characterized were the surface recombination velocity (SRV) and the Drude reflectance coefficient (DRC), although rough estimates of the SRV were obtained from a.c. photocurrent measurements, and the DRC could be obtained, to first order, from theory. Also, the pump radius was not precisely known because the pump spot-size was varied by adjusting the position of the focussing lens. Thus, for a given crystalline sample, the first step of the fitting procedure was to estimate the SRV and the DRC; then, the pump radius was varied until the RMS difference between the experimental PMOR amplitude curve and its corresponding theoretical curve was minimized. It should be noted that when the above fitting routine was carried out, the amplitude data were scaled so that the theoretical and experimental curves would coincide at the lowest modulation frequency; this procedure ensured that the "shape" of the fitted curve would match the experimental curve, but it did not take into account the absolute theoretical and experimental magnitudes.

Next, in order to ensure that the experimental and theoretical PMTR amplitude curves would agree absolutely, and not just in terms of shape, the SRV and DRC were adjusted until the absolute theoretical and experimental magnitudes were similar; note that every time the SRV or the DRC was altered, the pump radius was also varied, in order to maintain a good "shape" fit.

Overall, the following criteria were used to obtain the ultimate curve-fitting parameters: 1) The theoretical and experimental PMTR(f) amplitude curves had to match in terms of shape. 2) The theoretical and experimental PMTR(f) phase curves had to match in both shape and in the absolute sense. 3) The theoretical and experimental PMTR(f) amplitude data had to match in an absolute sense, and not just in terms of shape. 4) The SRV had to take on a value which was consistent with photocurrent data, and the DRC had to be within a factor of five of the theoretical value.

The above fitting procedure for the crystalline samples was found to provide theoretical PMTR amplitude and phase curves which corresponded well with the experimental data, both absolutely and in terms of shape. With respect to the uniqueness of a given set of fitting parameters (pump radius, SRV, and DRC), due to the fact that the fitting regime involved a stringent set of objective and subjective criteria which were difficult to satisfy simultaneously, it cannot be stated that a chosen set of fitting parameters was absolutely unique; on the other hand, since the parameters were allowed to vary over a wide range, it is believed that the chosen fitting parameters are within $50 \%$ of their "true" values, and that the relative size of the thermal and Drude signal components determined from the fitting exercise are accurate.

With respect to the disordered samples, the modelling of the PMTR signal was much more complicated than for the crystalline materials, due to the fact that there appeared to be a signal component related to the band-filling of localized gap states, while the Drude effect seemed to be negligible. Fortuitously, since the thermal and band-filling components were expected to have a very different frequency response, it was possible to determine the relative size of these two components from the PMTR(f) data, although it was not possible to extract meaningful carrier transport properties from the PMTR(f) curves. In order to obtain PMTR(f) curvefits for the disordered materials, the thermal conductivity and the effective carrier transport properties of the damage layer were varied until the experimental amplitude and phase curves matched the theoretical data both absolutely, and in terms of shape. Now, the results of the PMTR(f) curve-fitting exercise will be discussed. 
For the crystalline germanium (c-Ge) sample, the thermal signal component was found to be dominant under all conditions (Fig. 1), but the small Drude (free-carrier) component was observed to increase in importance as the modulation frequency and pump spot-size were increased. The c-Ge PMTR signal amplitude was a linear function of the absorbed pump power over a wide range, and no transient effects were observed.

For the implanted germanium (im-Ge) sample, the thermal component was significant, but there was no significant Drude signal (Fig. 2); instead, there was a signal which was hypothesized to be related to the band-filling of localized gap-states [4-6]. For the im-Ge sample, the trapped-carrier signal component was discovered to be very sensitive to the type of gas in contact with the sample; for instance, the PMTR signal was found to change considerably when the gas surrounding the sample was changed from dry oxygen to wet oxygen. The im-Ge PMTR signal amplitude was a linear function of the absorbed pump power, but saturation and transient phenomena were observed at higher excitation levels.

For the crystalline silicon materials, the thermal component was found to dominate at low frequency, while the Drude component became quite significant near $1 \mathrm{MHz}$, especially for the larger pump spot-size $(23.3 \mu \mathrm{m})$. For example, Fig. 3 displays a PMTR(f) curve obtained for the c-Si1 sample; note that the Drude signal component is quite large. In this figure it appears that the relative size of the Drude component does not vary significantly with respect to the total PMTR signal. In fact, the relative size of the Drude component grows as the frequency is increased, but this is not indicated in Fig. 3 because this figure does not take into account the vectorial character of the Drude and thermal PMTR components. The change in the nature of the PMTR signal is more evident in the phase data, which is not presented here. Furthermore, a power-law $\left(1^{1.5}\right)$ dependence of the PMTR signal upon the pump intensity was observed for the c-Si1 material at $10 \mathrm{kHz}$, and this indicates that non-linear (Auger) recombination effects were possibly of some importance [7]; this idea was confirmed by various theoretical calculations. The c-Si2 specimen also showed both thermal and Drude character (Fig. 4), but the Drude component was less significant than for the non-implanted c-Si1 sample; this result was attributed to a higher surface recombination velocity for the c-Si2. Note that the c-Si2 showed linear amplitude behaviour when the pump intensity was varied.

It is interesting to consider that the c-Si3 sample displayed certain non-ideal behaviourial traits which were not present for the other two crystalline silicon materials. For example, this sample had a highly supra-linear intensity dependence $\left(I^{2.25}\right)$, and saturation/transient effects were observed for high pump powers. This anomalous behaviour was attributed to a surface damage layer with a large density of active surface states [1].

Finally, for the amorphous silicon thinfilm (a-Si), as was found for the im-Ge sample, there were two important signal components, one thermal, and one related to the presence of trapped carriers.

In summary, the fitting of PMTR(f) data to a theoretical model has enabled us to separate the thermal and Drude signal components for crystalline $\mathrm{Ge}$ and $\mathrm{Si}$, and to identify the new trapped-carrier signal component for the im-Ge and a-Si samples.

\section{REFERENCES}

[1] Opsal J., Taylor M.W., Smith W.L., and Rosencwaig A., J. Appl. Phys. 61 (1987) 240-248.

[2] Dearnaley G., Freeman J.H., Nelson R.S., and Stephen J., Ion Implantation (North-Holland, Amsterdam, 1973) Appendix 3.

[3] Neuberger M., Group IV Semiconducting Materials (Plenum, New York, 1971).

[4] Chen L., Tauc J., and Vardeny Z., Phys. Rev. B 39 (1989) 5121-5127.

[5] Vardeny Z., Zhou T.X., Stoddart H.A., and Tauc J., Solid State Commun. 65 (1988) 1049-1053.

[6] Liu H., Pfost D., and Tauc J., Solid State Commun. 50 (1984) 987-990.

[7] Forget B.C., Fournier D., and Guse' ' .E., Appl. Phys. Lett. 61 (1992) 2341-2343. 


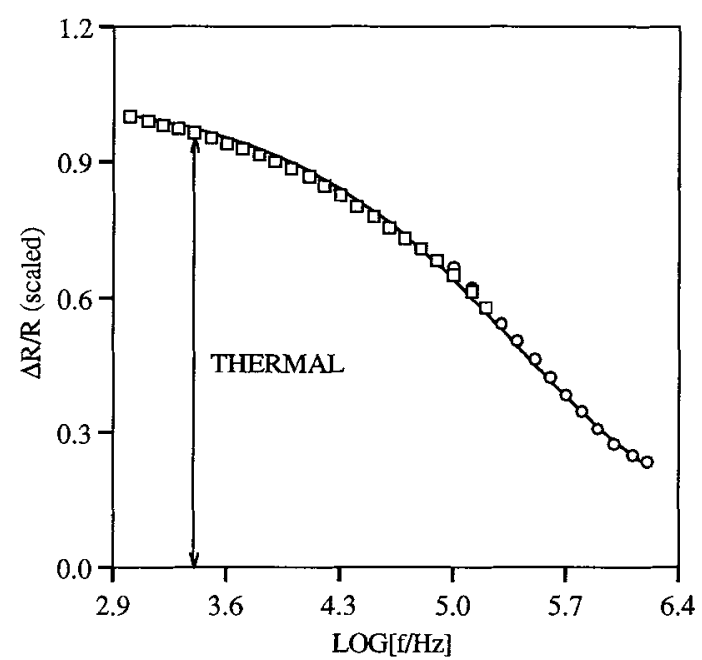

Fig. 1 - PMTR amplitude vs. frequency for c-Ge. The solid line is the theoretical fit, and the squares/circles are experimental data. The fitted $1 / \mathrm{e}$ pump radius is $7.5 \mu \mathrm{m}$, and the nominal value is $5.7 \mu \mathrm{m}$.

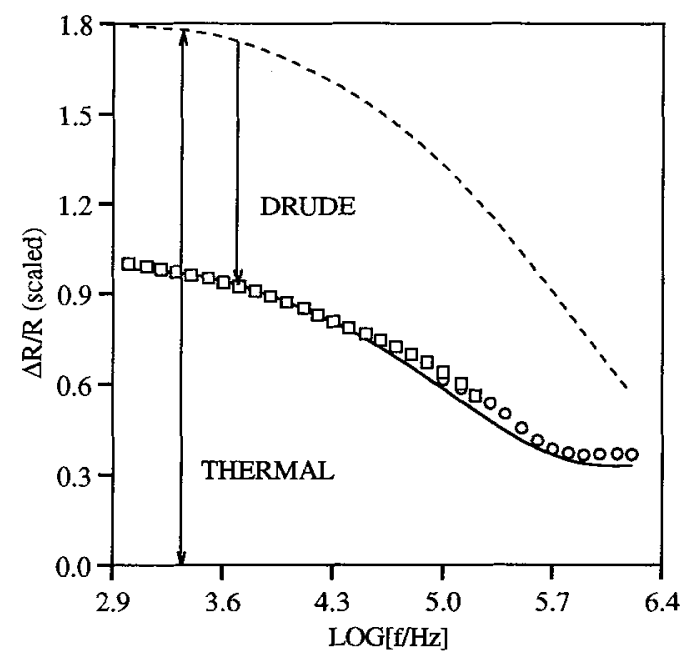

Fig. 3 - PMTR amplitude vs. frequency for c-Si1. The solid line is the theoretical fit, the dashed line is the thermal component from theory, and the squares/circles are experimental data. The fitted 1/e pump radius is 7.0 $\mu \mathrm{m}$, and the nominal value is $5.7 \mu \mathrm{m}$.

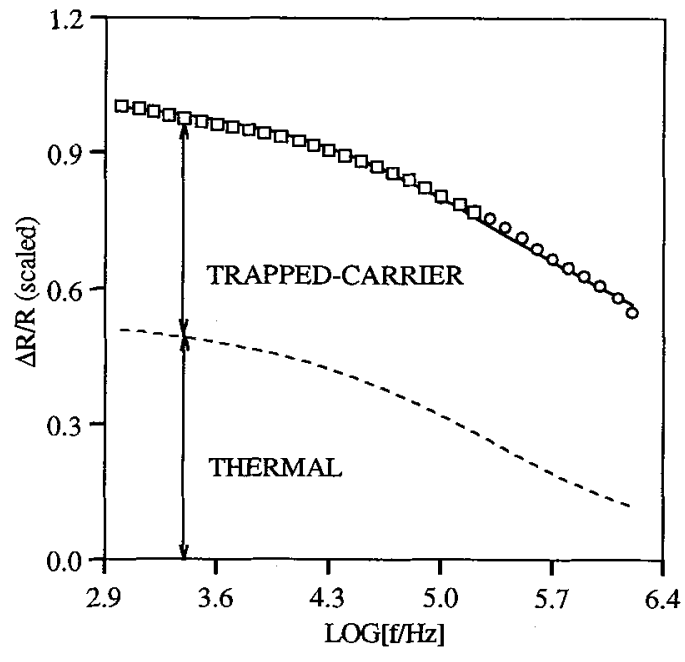

Fig. 2 - PMTR amplitude vs. frequency for im-Ge. The solid line is the theoretical fit, the dashed line is the thermal component from theory, and the squares/circles are experimental data. The fitted 1/e pump radius is 8.7 $\mu \mathrm{m}$, and the nominal value is $5.7 \mu \mathrm{m}$.

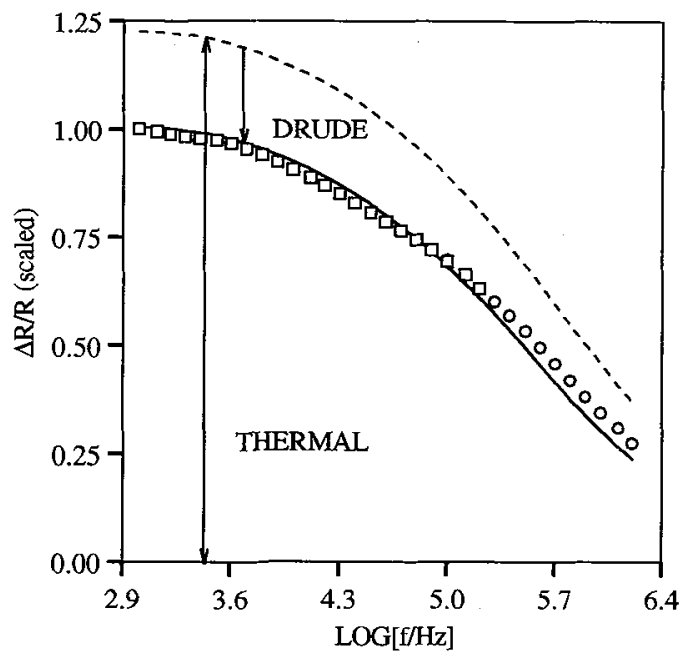

Fig. 4 - PMTR amplitude vs. frequency for $\mathrm{c}-\mathrm{Si} 2$. The solid line is the theoretical fit, the dashed line is the thermal component from theory, and the squares/circles are experimental data. The fitted $1 / \mathrm{e}$ pump radius is 7.1 $\mu \mathrm{m}$, and the nominal value is $5.7 \mu \mathrm{m}$. 